\title{
Personalized Models of Internalizing Symptoms for Women with Social Anxiety Disorder and Depression
}

\author{
Marilyn L. Piccirillo and Thomas L. Rodebaugh
}

Department of Psychological and Brain Sciences, Washington University in St. Louis

\begin{abstract}
Author note
Marilyn L. Piccirillo and Thomas L. Rodebaugh, Department of Psychological and Brain Sciences, Washington University in St. Louis, St. Louis, MO 63130.

Marilyn L. Piccirillo was supported by a National Research Service Award F31 MH 115641 from the National Institute of Mental Health. The authors do not have any conflicts of interest to declare. A preprint of this manuscript has been posted online prior to peer-review. Correspondence concerning this article should be addressed to Marilyn Piccirillo, Department of Psychological and Brain Sciences, Washington University in St. Louis, Campus Box 1125, St. Louis, MO 63130. Email: piccirillom@wustl.edu.
\end{abstract}




\begin{abstract}
Social anxiety disorder (SAD) constitutes an important risk factor for major depressive disorder (MDD) and women are at greater risk for both disorders and their comorbidity. Despite much research examining risk factors for MDD specifically, there is limited research evaluating how individuals with SAD transition into depressive episodes. Clinical and theoretical evidence suggests that each individual may exhibit a unique personalized pattern of risk factors. These idiographic patterns may contradict group-level findings. In this study, women $(N=35)$ with SAD and a current or past major depressive episode completed ecological sampling of their mood and emotional experience five times a day for a month via a smartphone application. These data were analyzed using idiographic analyses to construct individual-level models of each woman's mood. A multilevel model was constructed to determine risk factors for group-level intra-daily sadness (i.e., depressed mood). Some group-level relationships were consistent with previous research; however, most women's models demonstrated few, and differing, risk factors for intra-daily sadness. We also examined the spread of individual-level estimates taken from group and idiographic models to determine the extent to which multilevel models can estimate individual-level effects. Implications for integrating results from idiographic methodology into existing theoretical models of psychopathology and clinical practice are discussed.
\end{abstract}

Keywords: Idiographic; Multilevel; Ecological sampling; Internalizing disorders 
Personalized Models of Internalizing Symptoms for Women with Social Anxiety Disorder and

\section{Depression}

Social anxiety disorder (SAD) and major depressive disorder (MDD) are, collectively, the most prevalent mental disorders afflicting young adults, leading to decreased social and economic potential (Acarturk et al., 2008; Kessler et al., 2005). Billions of healthcare dollars are spent each year on morbidity associated with these disorders, and even more is estimated to be lost in workplace productivity, constituting an significant public health burden (Greenberg et al., 2003; Kessler et al., 2011; Kessler, Merikangas, \& Wang, 2007). Researchers have long demonstrated that young adults with SAD are especially vulnerable to develop MDD, which often arises earlier and is of greater severity than MDD for individuals without SAD (Dalrymple \& Zimmerman, 2011; Kessler, 1997; Pini et al., 1997; Stein et al., 2001). Furthermore, women are at greater risk for internalizing disorders, including SAD and depression (Leach et al., 2008; Nolen-Hoeksema \& Girgus, 1994).

The Cumulative Interpersonal Risk model developed by Epkins and Heckler (2011) describes a diathesis-stress model of SAD leading to MDD. This model suggests that individuals with SAD may be at especially high risk for developing MDD due to shared genetic, affective, and cognitive factors (Cummings et al., 2014; Epkins \& Heckler, 2011). Affective factors, coupled with negative self-referential biases and cognitive strategies put individuals at greater risk of developing internalizing disorders (Cummings et al., 2014; Epkins \& Heckler, 2011). Thus, the transition from this risk state into MDD is hypothesized to result from maladaptive social processes and stressful interpersonal outcomes, such as withdrawal and social rejection (Starr et al., 2014).

Numerous studies focusing on the etiology of and risk factors for SAD and MDD have 
provided support for theoretical models of comorbidity (Hirshfeld-Becker et al., 2008; HirshfeldBecker, 2010; Rapee \& Spence, 2004; Rudolph, Flynn, \& Abaied, 2008). However, despite decades of research on etiological and risk factors for these disorders, there is limited research on methods for preventing or effectively treating this comorbidity (Epkins \& Heckler, 2011). An additional, and perhaps a more significant, limitation may be the methodology used in previous research. Clinical psychologists have historically used primarily cross-sectional or longitudinal between-subjects (group-level) designs to identify risk factors for MDD. Arguably, risk factors identified at the group-level may be non-significant, or even protective, for specific individuals within the group (Fisher, Medaglia, \& Jeronimus, 2018; Molenaar, 2004) thus limiting treatment and prevention efforts that are based on these group-level risk factors.

The use of person-specific methodology allows clinical scientists to examine whether risk factors elucidated from group-level designs manifest on the individual-level and if so, how these patterns differ between individuals. In this way, each model represents the results of an individual-specific study (Wichers, 2014). This methodology complements traditional groupbased designs and may provide insight into idiographic, individual-specific factors that characterize symptomatology. Furthermore, pairing multilevel and idiographic designs allows researchers to accurately model both inter- and intra-individual variability and demonstrate how group-level relationships differ from individual-specific trends. Results from idiographic analyses may assist with personalizing clinical assessment and intervention, by facilitating the selection of more targeted empirically supported interventions based on person-specific findings (Fisher et al., 2019; Piccirillo, Beck, \& Rodebaugh, 2019).

In this study, we constructed idiographic models of internalizing symptoms for women using ecological sampling data collected multiple times a day. We constructed individual and 
group-level models in tandem to examine the concordance across model levels. We recruited women diagnosed with current SAD and either a current major depressive episode or at least one past major depressive episode, allowing us to examine the individual-specific relationships between affective and behavioral factors that influence intra-day fluctuations in sadness for a group of women at higher risk for internalizing symptoms. We hypothesized that maladaptive behaviors, such as social avoidance, or affective states, such as anxiety, would be the strongest risk factors for intra-day sadness and that results would differ across women. Furthermore, we constructed a multilevel model to examine risk factors for sadness on the group-level. We hypothesized that although women may share some pathways in common with the group model, no one individual model would demonstrate the precisely the same structure as the group-level model.

\section{Methods}

\section{Participants}

As women are at greater risk for internalizing disorders, including SAD and depression (Leach et al., 2008; Nolen-Hoeksema \& Girgus, 1994), we recruited cis-gender women ${ }^{1}(N=35)$ from the general university community. Most women $(n=26)$ were enrolled in undergraduate courses; however, others were graduate students or employees $(n=9)$. All women completed a diagnostic clinical interview as part of their participation in a separate, parent study. Results from the diagnostic interview were used in recruitment for this study and self-report measures were used for examining psychometric properties of ecological sampling items. Those who met criteria for current SAD and a current $(n=6)$ or past major depressive episode $(n=29)$ were invited and enrolled in this study. Importantly, studies using related methodologies have shown

\footnotetext{
${ }^{1}$ Only women who self-identified as cis-gender were recruited, as we were not able to study the effects of sex or gender due to the limited sample size.
} 
that there is still significant variation in affect among individuals who are clearly depressed (Peeters et al., 2006). Ages ranged from $18-37$ years $(M=21.37$ years, $S D=5.20)$, and approximately half of the women identified as White $(51.43 \%)$. There was considerable racial and ethnic diversity as $28.57 \%$ women identified as East Asian, $17.14 \%$ women identified as Black, and 5.71\% women identified as Hispanic.

\section{Measures}

\section{MINI Interview - 5}

The MINI-5 is a structured interview that assesses psychological disorders and was used in the parent study to assess clinical disorders. Inter-rater reliability was assessed using blinded independent raters. Inter-rater reliability was good for SAD $(\kappa=.73)$ and excellent for a MDE $(\kappa$ $=.83)$.

\section{Ecological Sampling Method (ESM) Items}

Daily ESM assessments consisted of 14 items that reflected constructs implicated in the Cumulative Interpersonal Risk Model (Epkins \& Heckler, 2011) and in the diagnostic criteria for an MDD, such as sadness, anxiety, irritability, and restlessness. Additionally, physiological or behavioral indicators, such as hunger, physical activity, and social avoidance that may play a role in predicting affect were also included. Participants rated all questions based on their presentmoment experience (e.g., Hektner, Schmidt, \& Csikszentmihalyi, 2007; Shiffman, Stone, \& Hufford, 2008). Items were administered using a 0 - 10 integer sliding scale, with 1-pt interval responses (Hektner et al., 2007). ${ }^{2}$ It was estimated that the entire survey took less than three minutes to complete at each assessment. To examine validity of the ESM items, each item was correlated with the closest theoretical self-report item measured at baseline in the separate study.

\footnotetext{
2There was an error in the scale creation of two ESM items that affected two participants in the sample. These participants used an 11-point 0 - 100 integer sliding scale instead of a 0 - 10 integer sliding scale. Responses from these two items were collapsed to form a 0 - 10 integer scale (i.e., a 70 was converted to a 7).
} 
Descriptions of these self-report measures are included in supplementary material and correlations are presented in Table 1. Overall correlations between ESM items and self-report baseline items ranged in effect size, suggesting fair construct validity. Correlations between ESM items were also examined and were consistent with hypothesized relationships in both magnitude and valence (i.e., strong negative correlation between Down and Happy; see Table 2).

\section{Procedure}

Eligible participants provided informed consent before enrolling and all study procedures were approved by the Institution Review Board. Participants underwent a brief training protocol administered by the first author to introduce the ESM items, how to use the ESM scale, and how to use the free smartphone app. ${ }^{3}$ ESM assessments were administered five times a day during a participant-selected 12-hour time range for 30 days (for a total of 150 assessments). Participants received weekly progress emails, which documented the number of surveys completed and the amount of compensation earned for the week.

At the end of the 30-day period, participants returned to the lab to complete the second session of the separate study. They were debriefed on the purpose of both studies and received a preliminary version of their personalized model using output generated from their data. Participants received monetary compensation in proportion to the number of ESM surveys they completed each day. Maximum compensation was $\$ 82.50$ for $100 \%$ completion of ESM surveys and a majority of participants completed over 100 surveys $\left(M_{\text {surveys }}=125.43, S D=19.26\right)$.

\section{Data Analytic Plan}

Prior to all study analyses, each participant's data was visually inspected and cleaned. Extraneous variables (e.g., timestamps) were removed and missing data was specified. 
Additionally, to prepare data for dynamic structure equation modeling (DSEM; Asparouhov et al., 2018; Muthén \& Muthén, 1998-2017), three rows of missing data were added between each day to account for the three assessments that would have been administered outside of the 12hour time range. The addition of these missing rows ensured that the model did not predict trends over overnight periods, which would violate the statistical assumptions of DSEM.

DSEM uses a structural equation modeling approach with Bayesian estimation to model direct inter-item relationships, as well as latent variables. Importantly, DSEM is able to directly model effects of time by adding these pathways to the structural equation model. DSEM models included autoregressive and cross-lagged pathways between ESM items. An autoregressive pathway represents the relationship between one variable at two different time points (i.e., $\mathrm{X}_{-1}$ predicting $\mathrm{x}$ ); whereas a cross-lagged pathway represents the relationship between two variables from one time point to the next (i.e., $\mathrm{x}_{-1}$ predicting $\mathrm{y}$ ). Mplus notifies the user of the number of iterations in a DSEM analysis that may contain some violations to stationarity, which assists the user in determining whether the individual's data meets the assumptions of time series analysis.

Before beginning DSEM analyses, we planned to conduct exploratory factor analyses (EFA), or p-technique, using each participant's data to determine whether latent variables should be modeled using DSEM (see Fisher, 2015 for an example of this method). Up to five factors were specified and output from parallel analyses, as well as cutoffs for standard fit indices were examined. If there was a simple factor structure, we planned to model this latent variable structure using DSEM. ${ }^{4}$ Ultimately, no participant's data evidenced simple structure so individual items were analyzed. Furthermore, to reduce Type I error, DSEM models included pathways for sadness predicted by all other ESM items and pathways for all ESM items

\footnotetext{
${ }^{4} \mathrm{~A}$ simple factor structure refers to a model structure in which there are a sufficient number of indicators loading onto each factor with low cross-loadings on the other factors (as described in Yang, 2005).
} 
predicted by time variables. This model was chosen because sadness was the primary ESM item of interest.

To construct individual DSEM models, an open lag 1 model was run without specifying iterations. After the model converged, successive models were run with twice as many iterations and the proportional scale reduction (PSR) factor was evaluated (Muthén, 2010). The model was considered to be final when at least two models converged with low, stable PSR (a cutoff of 1.01 was used). When necessary, commands to prune iterations (Asparouhov, 2014) and noninformative priors (L. Muthén, personal communication, 12/13/2017) were used to achieve model convergence.

To model group-level relationships, multilevel DSEM (ML-DSEM) was used (Hamaker et al., 2018) to model lag 1 autoregressive and cross-lagged relationships. We constructed a model that included the random within-level auto-regressive effects for sadness, as well as the cross-lagged effects of sadness predicted by all ESM items. We also regressed all ESM items on time variables and random error terms. Means and variance of each parameter from the within level were included on the between-level. ML-DSEM also generates individual-level estimates for each pathway using information obtained in the generation of the group-level model. These estimates were used in comparison with the individual-level estimates generated from each idiographic DSEM (see below for further discussion). Mplus, version 8.2 (Muthén \& Muthén, 1998-2017) was used to conduct the EFA and DSEM analyses. Lag 2 models were also constructed for individual-level and multilevel models. Results were largely similar to lag 1 models and are included in the supplementary material. We also examined multilevel and individual-level DSEM models for multicollinearity. Multicollinearity exhibited minor effects on two women's individual level models and these results are discussed in supplementary material. 
To compare overlap between idiographic and multilevel models, we examined the 95\% credible intervals for each pathway to determine whether the interval did not contain 0 , thus suggesting a statistically significant pathway. We compared the number of statistically significant pathways that were demonstrated in the multilevel model with the number of statistically significant pathways that were represented in the individual-level models and calculated the percentage of such individual-level pathways that were shared with the multilevel model. To assist with data visualization, we constructed density plots of the standardized individual-level estimates computed by the multilevel model compared with the standardized estimates produced in each individual-level model for each pathway predicting daily sadness. These graphs serve to visualize whether the individual-level estimates from multilevel DSEM versus idiographic DSEM models reflected similar distributions. Plots were generated using R, including the Mplus Automation package, version 0.7-3 (Hallquist \& Wiley, 2018) and the ggplot2 package, version 3.1.0 (Wickham, 2016). Code for all analyses is included in supplementary material.

\section{Power Analyses}

There were no previous simulation studies to suggest the sample size needed in order to provide sufficient power for these proposed analyses at the time of study design. However, the study design allowed for the collection of over 100 ESM time points, which was in keeping with previous studies of this nature that treat each individual as a single study for individual-level analysis (Wichers, 2014).

\section{Results}

\section{Multilevel Model}

The multilevel model converged with stable, low PSR across successive models with 
increasing iterations. Multilevel effects with a $95 \%$ credible interval that did not include 0 can be seen in Figure 1. Overall, there were significant effects of time, such that feeling down increased over the month. Additionally, there was a significant autoregressive effect of feeling down, such that feeling down at one time point predicted feeling more down three hours later. Feeling lonely, avoiding social situations, and more physical activity since the last assessment were all risk factors for feeling more down three hours later; whereas, feeling calm was a protective factor for feeling down.

\section{Individual-level Models}

Examining each woman's individual-level model revealed considerable variability. Only a minority of women $(n=14,56.00 \%)$ had models that demonstrated at least one statistically significant autoregressive or cross-lagged effect (see Figure 2). Of these fourteen individuals, two women (IDs 14 and 20) demonstrated models that reflected one directed effect, such that for these two women, their feelings of sadness increased over the month. Likewise, there were two women (IDs 13 and 22) whose models reflected one significant effect - an autoregressive effect of down - suggesting that for these two individuals, their feelings of sadness were predicted by higher levels of sadness at the previous time point. The remaining ten women exhibited varying pathways (significant pathways demonstrated in Figure 1).

\section{Comparison Of Estimates From Multilevel Models And Individual-level Models}

Of the six significant pathways indicated in the group-level model, women had few pathways in common with the multilevel model, $(M=0.37, S D=0.65$, range $=0-2)$. The most frequent congruent pathway was the significant effect of down increasing across the month $(n=$ 4, $11.43 \%)$, followed by the significant positive autoregressive effect of feeling down $(n=3$, $8.57 \%$ ); however, these pathways did not occur with great frequency. Furthermore, there were 
three instances of pathways that were incongruent in valence with the group level model (e.g., the group-level model had a positive estimate, whereas the individual-level model contained a negative estimate). One woman demonstrated a model in which her sadness decreased (rather than increased) over the course of the month and social avoidance led to decreased (rather than increased) sadness later on. For a second woman, feeling lonely predicted less sadness later on.

Density plots of estimates from the multilevel model were graphed alongside the density plots of individual-level estimates obtained from the multilevel model output. The estimates from the down autoregressive pathway appear to reflect two distributions with different means. However, the estimates from all other cross-lagged pathways appear to represent a similar distribution with individual-level estimates reflecting a larger spread of distribution compared to the multilevel estimates. Furthermore, the difference in spread of distributions between group and individual-level estimates did not appear to depend on number of observations. The amount of overlap in multilevel versus individual-level estimates is demonstrated in Figure 2.

\section{Discussion}

We constructed idiographic models of internalizing symptoms for women with SAD and depression and demonstrated that, on the individual level, predictors of sadness differed across women. Moreover, few patterns from the group-level model were reflected in any one individual-level model. For example, there was minimal concordance between the statistically significant pathways demonstrated in the multilevel and individual-level DSEM models. Additionally, the individual-level estimates taken from multilevel and individual-level DSEM models roughly reflected similar distributions, with one exception. The two distributions for the autoregressive pathway of down differed substantially based on the source of the individual-level estimates (i.e., multilevel versus idiographic model). 
Results from the multilevel model are largely in keeping with previous literature. For example, feelings of loneliness, feeling less calm, and greater social avoidance predicted feeling down three hours later. These findings are consistent with the Cumulative Interpersonal Risk Model, which highlight the unique associations of loneliness with both social anxiety and depression for adolescent women as a maintenance factor for depression. Similarly, social avoidance and isolation are known maintenance factors of internalizing symptoms (Epkins \& Heckler, 2011). Additionally, there were clear effects of time. That is, on average, feelings of sadness increased systematically over the course of the month and across time points. Interestingly, and counterintuitively, greater physical activity since the last time point predicted increased feelings of sadness later on.

These findings are somewhat inconsistent with theories of behavioral activation, which suggests that activities that are associated with positive affect and accomplishment have an ameliorative effect on mood. For example, there were no significant directed multilevel pathways and few idiographic pathways between feeling down and feeling happy, pleased with one's experience, or accomplished over the course of three hours. Furthermore, in the multilevel model, amounts of physical activity since the last time point positively predicted feelings of sadness, which is in contrast to previous studies suggesting that physical activity may be beneficial for mood (Peluso \& Guerra De Andrade, 2005).

It is unclear why engagement in physical activity would predict increased feelings of sadness. Previous literature describing the acute and beneficial effects of physical activity on mood have largely defined physical activity as aerobic exercise or running (Yeung, 1996). In this study, participants were encouraged to report on any type of physical activity engaged in since the last time point, and therefore were not restricted to aerobic activity, limiting generalizability 
of these results to previous literature. Furthermore, literature on behavioral activation underscores the positive reinforcing nature of physical activity that is consistent with the individual's values (Lejuez \& Hopko, 2013). Again, as there were no specifications on how women defined physical activity, women may have been engaging in physical activity that was inconsistent with how physical activity has been measured in previous studies.

Results comparing group and individual level models were consistent with our hypothesis that no one individual model would demonstrate precisely the same model structure as the grouplevel model. Interestingly, shared pathways were more likely to be congruent (i.e., of similar valence) than incongruent (i.e., of different valence). However, no one individual shared all significant pathways with the group-level model (number of statistically significant pathways overlapping with the multilevel model ranged from 0 - 2). Systematic increases in feeling down over the course of the month $(n=4)$, as well as a positive autoregressive effect for feeling down $(n=3)$ were the most common multilevel pathways evident in individual DSEM models; however, these pathways were only significant in a few models. Notably, three of the four women whose individual-level model demonstrated a systematic effect of time over the month actually completed the study during the summer, suggesting that this effect was not primarily due to the nature of the academic semester. Given that few studies have examined multilevel and individual-level DSEM models in tandem, these results necessitate careful consideration to determine which model provides the most useful information regarding individuals.

Examining the distribution spread of individual-level pathways taken from the idiographic and multilevel models revealed an interesting pattern. The distributions of individual-level estimates were largely similar regardless of model source (i.e., idiographic versus multilevel model), with one exception. That is, when examining the autoregressive path, 
there appeared to be two distinct distributions suggesting that the multilevel model predicted autoregressive paths for sadness differently than the idiographic DSEM models. It is unclear why these estimates differed in their distributions and thus, it is an open question as to whether the multilevel or idiographic DSEM models in this study best estimates the down autoregressive relationship. A previous group of simulation studies conducted by Liu (2017) suggested that there may be specific situations in which multilevel autoregressive models outperform idiographic autoregressive models. However, these situations are unlikely to apply to this sample, and thus, in the absence of additional simulation data modeling we cannot determine whether the multilevel model is any more accurate than the individual-level DSEM model in modeling autoregressive pathways. Future research is needed to systematically examine the conditions under which multilevel models accurately model individual-level effects.

The distribution for the cross-lagged pathways from each model source were largely similar, although estimates from idiographic models had considerably larger spread suggesting that the discrepancy between multilevel and idiographic estimates may have resulted from higher variance across the idiographic models (Figure 2). This is consistent with a previous studies that have demonstrated that variance around a mean estimate and interquartile range, respectively, are typically large on the individual-level (Fisher et al., 2018; Zimmerman et al., 2019). It may be that the higher variance seen across idiographic models is actually higher error variance; however, these findings did not appear to change based on the participant's number of completed assessments (see Figure 2). Further research is needed to determine whether this discrepancy was due to limited power (i.e., higher error variance) in the idiographic DSEM model or because individuals with fewer time points were pulled towards the mean in a multilevel model. 
This study demonstrated key strengths in that idiographic models were constructed in tandem with a group-level model, allowing us to examine differences in concordance between the two sources of estimates, furthering our understanding of how individuals differ from the group. Furthermore, this study used the latest time series methodology available for modeling longitudinal intraindividual variability. DSEM methods improve upon previous methodology in that we can identify some violations to stationarity and note when data does not meet the assumptions of the given time series approach.

There were several limitations to this study, two of which we will highlight here as they temper our conclusions and call for future research. One of these limitations is the time scale used to examine affective and behavioral experiences. Results from this study reflect relationships demonstrated when items are sampled every three hours. However, it is likely that if we were to have assessed these items on a different schedule, a different pattern of results may have emerged, Future research is sorely needed to examine the extent to which idiographic differences influence the time course of psychological experiences. Secondly, our evaluation of the psychometric properties of the ESM items created for this study was limited. Despite recent steps to improve the selection of ESM items broadly (e.g., the Experience Sampling Item Repository, esmitemrepository.com) and calls for increasing focus on testing the psychometric properties of ESM items (see Trull \& Ebner-Priemer, 2020; Wright \& Zimmermann, 2018), there are few direct guidelines available to assist researchers in testing the validity of ESM items. In this study, we examined the correlations between ESM items and theorized self-report item matches at baseline and demonstrated variable effect sizes. Arguably these comparisons may not be appropriate given the differences in timeline of assessment (e.g., trait measures versus state measures referring to a specific timeframe) and differences in the nature of constructs being 
measured (e.g., sense of present-moment accomplishment versus feelings of past failure). Thus, the presented correlations seem questionable as evidence (whether for or against) of construct validity. The contemporaneous correlations present a more compelling argument that ESM items performed as hypothesized; however, future work is needed to support the psychometric evaluation of ESM items used in group-level research and to delineate the effect of idiographic differences on psychometric properties.

These findings raise important questions about how to integrate results from intensive longitudinal and idiographic work with existing theoretical models of comorbid internalizing disorders, given that these theories have largely been developed using cross-sectional or grouplevel longitudinal research. For example, although loneliness and social avoidance were predictors of intra-daily sadness, in keeping with the Cumulative Interpersonal Risk Model, several additional variables implicated in this model, such as irritability, anxiety, feeling accomplished, and feeling pleased with ones' experience, were not. Furthermore, very few pathways predicting sadness were consistent across idiographic models. These results clearly underscore the effect of social behaviors and relationships in maintaining comorbid internalizing disorders. Although previous research has highlighted the role of irritability and positive affect as features of comorbid SAD and depression (Langer et al., 2019), this research was cross-sectional in nature and thus, these relationships may not be present when measured longitudinally.

Furthermore, most idiographic models were sparse in nature and some significant relationships were incongruent in valence. For example, one woman's levels of loneliness predicted lower (rather than higher) depression. These findings suggest that if individual risk models were to be used in treatment planning, it would be useful for the clinician to note whether the client's model reflects a significant pathway between loneliness and sadness in the 
hypothesized direction, if the pathway is reflected at all. One possibility is that this woman was already using effective coping strategies to manage feelings of loneliness. For example, she may feel lonely when spending time alone; however, spending time alone also may have facilitated mood-regulation activities (e.g., meditation) that decreased her sadness later on. However, future research is needed to determine to what extent results from multilevel and idiographic models can be used to improve upon our existing theories of internalizing disorders.

The comparison between the estimates generated from the idiographic models and the multilevel model also provides avenues for future research. For example, the multilevel model may be able to more adequately model intraindividual variability due to greater statistical power, while also requiring fewer observations for each individual (Schultzberg \& Muthén, 2018). Furthermore, results have demonstrated moderate to high correlations between parameters from multilevel and individual-level models (Rodebaugh, Piccirillo, Frumkin, Gerull, Kallogjeri, \& Piccirillo, 2019). That is, provided multilevel models are able to accurately estimate individuallevel effects, it may be more feasible to use ML-DSEM to estimate individual-level relationships, rather than estimating idiographic models in parallel.

The issues with intensive longitudinal designs and idiographic methodology provide clear avenues for future research to determine whether multilevel models accurately describe individuals within the group. Future research using intensive longitudinal methods within experimental designs are needed to help determine whether group-level results adequately describe individuals or if, in contrast, individual-level models provide superior predictive power. For example, consider a group-level model in which social stress predicts future depressive symptoms, but some individuals show the opposite pattern in their individual models. Systematically varying social stress (e.g., in an experimental task) and determining whether the 
group or individual model better predicts an individual's resulting trajectory of depressed mood would help clarify whether differential findings in individual models serve as signal or noise. Pursuing these issues in future work will provide evidence to answer key questions in the field of idiography - that is, do group-level models of psychopathology ever fully describe individuals within the group, and if so, when, and under what circumstances? Results from future work can inform the extent to which these findings can be used in clinical and applied settings. 


\section{References}

Acarturk, C., de Graaf, R., van Straten, A., ten Have, M., \& Cuijpers, P. (2008). Social phobia and number of social fears, and their association with comorbidity, health-related quality of life and help seeking. Social Psychiatry and Psychiatric Epidemiology, 43(4), 273-279. https://doi.org/10.1007/s00127-008-0309-1

Asparouhov, T. (2014). Specifying burn in for Bayes. Mplus Discussion. http://www.statmodel.com/discussion/messages/11/20726.html?1547519147

Asparouhov, T., Hamaker, E. L., \& Muthén, B. (2018). Dynamic structural equation models. Structural Equation Modeling: A Multidisciplinary Journal, 25(3), 359-388. https://doi.org/10.1080/10705511.2017.1406803

Cummings, C. M., Caporino, N. E., \& Kendall, P. C. (2014). Comorbidity of anxiety and depression in children and adolescents: 20 years after. Psychological Bulletin, 140(3), 816845. https://doi.org/10.1037/a0034733

Dalrymple, K. L., \& Zimmerman, M. (2011). Age of onset of social anxiety disorder in depressed outpatients. Journal of Anxiety Disorders, 25(1), 131-137.

https://doi.org/10.1016/j.janxdis.2010.08.012

Epkins, C. C., \& Heckler, D. R. (2011). Integrating etiological models of social anxiety and depression in youth: Evidence for a Cumulative Interpersonal Risk Model. Clinical Child and Family Psychology Review, 14(4), 329-376. https://doi.org/10.1007/s10567-011-01018

Fisher, A. J. (2015). Toward a dynamic model of psychological assessment: Implications for personalized care. Journal of Consulting and Clinical Psychology, 83(4), 825-836. https://doi.org/10.1037/ccp0000026 
Fisher, A. J., \& Bosley, H. G. (2015). Personalized assessment and treatment of depression. Current Opinion in Psychology, 4(4), 67-74. https://doi.org/10.1016/j.copsyc.2015.03.031

Fisher, A. J., Medaglia, J. D., \& Jeronimus, B. F. (2018). Lack of group-to-individual generalizability is a threat to human subjects research. Proceedings of the National Academy of Sciences of the United States of America, 115(27), E6106-E6115. https://doi.org/10.1073/pnas.1711978115

Greenberg, P. E., Kessler, R. C., Birnbaum, H. G., Leong, S. A., Lowe, S. W., Berglund, P. A., \& Corey-Lisle, P. K. (2003). The economic burden of depression in the United States. The Journal of Clinical Psychiatry, 64(2), 1465-1475. https://doi.org/10.4088/JCP.v64n1211

Hallquist, M. N., \& Wiley, J. F. (2018). Mplus Automation: An R package for facilitating largescale latent variable analyses in Mplus. Structural Equation Modeling, 25(4), 621-638. https://doi.org/10.1080/10705511.2017.1402334

Hamaker, E. L., Asparouhov, T., Brose, A., Schmiedek, F., \& Muthén, B. (2018). At the frontiers of modeling intensive longitudinal data: Dynamic structural equation models for the affective measurements from the COGITO study. Multivariate Behavioral Research, 53(6), 820-841. https://doi.org/10.1080/00273171.2018.1446819

Hektner, J. M., Schmidt, J. A., \& Csikszentmihalyi, M. (2007). Experience sampling method: Measuring the quality of everyday life. SAGE Publications Inc.

Hirshfeld-Becker, D., Micco, J., Henin, A., Bloomfield, A., Biederman, J., \& Rosenbaum, J. (2008). Behavioral inhibition. Depression and Anxiety, 25(4), 357-367. https://doi.org/10.1002/da.20490

Hirshfeld-Becker, D. R. (2010). Familial and temperamental risk factors for social anxiety disorder. New Directions for Child and Adolescent Development, 2010(127), 51-65. 
https://doi.org/10.1002/cd.262

Kessler, R. C. (1997). Prevalence, correlates, and course of minor depression and major depression in the National Comorbidity Survey. Journal of Affective Disorders, 45(1-2), 19-30. https://doi.org/10.1016/S0165-0327(97)00056-6

Kessler, R. C., Aguilar-Gaxiola, S., Alonso, J., Chatterji, S., Lee, S., Ormel, J., Ustün, T. B., \& Wang, P. S. (2011). The global burden of mental disorders: An update from the WHO World Mental Health (WMH) surveys. Epidemiologia e Psichiatria Sociale, 18(1), 23-33. https://doi.org/10.1017/S1121189X00001421

Kessler, R. C., Berglund, P., Demler, O., Jin, R., Merikangas, K. R., \& Walters, E. E. (2005). Lifetime prevalence and age-of-onset distributions of DSM-IV disorders in the National Comorbidity Survey Replication. Archives of General Psychiatry, 62, 593-602. https://doi.org/10.1001/archpsyc.62.6.593

Kessler, R. C., Merikangas, K. R., \& Wang, P. S. (2007). Prevalence, comorbidity, and service utilization for mood disorders in the United States at the beginning of the twenty-first century. Annual Review of Clinical Psychology, 3, 137-158. https://doi.org/10.1146/annurev.clinpsy.3.022806.091444

Langer, J. K., Tonge, N. A., Piccirillo, M. L., Rodebaugh, T. L., Thompson, R. J., \& Gotlib, I. H. (2019). Symptoms of social anxiety disorder and major depressive disorder: A network perspective. Journal of Affective Disorders, 243, 531-538. https://doi.org/https://doi.org/10.1016/j.jad.2018.09.078

Leach, L. S., Christensen, H., Mackinnon, A. J., Windsor, T. D., \& Butterworth, P. (2008). Gender differences in depression and anxiety across the adult lifespan: The role of psychosocial mediators. Social Psychiatry And Psychiatric Epidemiology, 43(12), 983-998. 
https://doi.org/10.1007/s00127-008-0388-z

Lejuez, C. W., \& Hopko, D. R. (2013). Brief Behavioral Activation (BA) Treatment Manual Revised. University of Maryland College Park.

Molenaar, P. C. M. (2004). A manifesto on psychology as idiographic science: Bringing the person back into scientific psychology, this time forever. Measurement, 2(4), 201-218. https://doi.org/10.1207/s15366359mea0204_1

Muthén, B., \& Muthén, L. (1998-2017). Mplus, version 8.0.

Muthén, Bengt. (2010). Bayesian analysis in Mplus: A brief introduction, version 3.0.

Nolen-Hoeksema, S., \& Girgus, J. S. (1994). The emergence of gender differences in depression during adolescence. Psychological Bulletin, 115(3), 424-443. https://doi.org/10.1037/00332909.115.3.424

Peeters, F., Berkhof, J., Delespaul, P., Rottenberg, J., \& Nicolson, N. A. (2006). Diurnal mood variation in major depressive disorder. Emotion, 6(3), 383-391. https://doi.org/10.1037/1528-3542.6.3.383

Peluso, M. A. M., \& Guerra De Andrade, L. H. S. (2005). Physical activity and mental health: The association between exercise and mood. CLINICS, 60(1), 61-70.

Piccirillo, M. L., Beck, E. D., \& Rodebaugh, T. L. (2019). A clinician's primer for idiographic research: Considerations and recommendations. Behavior Therapy, 50(5), 938-951. https://doi.org/10.1016/j.beth.2019.02.002

Pini, S., Cassano, G. B., Simonini, E., Savino, M., Russo, A., \& Montgomery, S. A. (1997). Prevalence of anxiety disorders comorbidity in bipolar depression, unipolar depression and dysthymia. Journal of Affective Disorders, 42(2-3), 145-153. https://doi.org/10.1016/S0165-0327(96)01405-X 
Rapee, R. M., \& Spence, S. (2004). The etiology of social phobia: Empirical evidence and an initial model. Clinical Psychology Review, 24(7), 737-767. https://doi.org/10.1016/j.cpr.2004.06.004

Rodebaugh, T. L., Piccirillo, M. L., Frumkin, M. R., Gerull, K. M., Kallogjeri, D., \& Piccirillo, J. F. (2019). Individual variation in tinnitus models as a potential predictor of intervention response [Manuscript submitted for publication]. Department of Psychological and Brain Sciences, Washington University in St. Louis.

Rudolph, K. D., Flynn, M., \& Abaied, J. L. (2008). A developmental perspective on interpersonal theories of youth depression. In B. L. Hankin \& J. R. Z. Abela (Eds.), Handbook of Depression in Children and Adolescents (pp. 79-102). Guilford Press.

Schultzberg, M., \& Muthén, B. (2018). Number of subjects and time points needed for multilevel time-series analysis: A simulation study of dynamic structural equation modeling. Structural Equation Modeling, 25(4), 495-515. https://doi.org/10.1080/10705511.2017.1392862

Shiffman, S., Stone, A. A., \& Hufford, M. R. (2008). Ecological momentary assessment. Annual Review of Clinical Psychology, 4, 1-32.

https://doi.org/10.1146/annurev.clinpsy.3.022806.091415

Starr, L. R., Hammen, C., Connolly, N. P., \& Brennan, P. A. (2014). Does relational dysfunction mediate the association between anxiety disorders and later depression? Testing an interpersonal model of comorbidity. Depression and Anxiety, 31(1), 77-86. https://doi.org/10.1002/da.22172

Stein, M. B., Fuetsch, M., Müller, N., Höfler, M., Lieb, R., \& Wittchen, H.-U. (2001). Social anxiety disorder and the risk of depression: A prospective community study of adolescents 
and young adults. Archives of General Psychiatry, 58(3), 251-256.

https://doi.org/10.1001/archpsyc.58.3.251

Trull, T. J., \& Ebner-Priemer, U. W. (2020). Ambulatory assessment in psychopathology research: A review of recommended reporting guidelines and current practices. Journal of Abnormal Psychology, 129(1), 56-63. https://doi.org/10.1037/abn0000473

Wichers, M. (2014). The dynamic nature of depression: a new micro-level perspective of mental disorder that meets current challenges. Psychological Medicine, 44(7), 1349-1360. https://doi.org/10.1017/S0033291713001979

Wickham, H. (2016). ggplot2: Elegant Graphics for Data Analysis. Springer-Verlag New York. Wright, A., \& Zimmermann, J. (2018). Applied ambulatory assessment: Integrating idiographic and nomothetic principles of measurement. November. https://doi.org/10.31234/osf.io/6qc5x

Yang, B. (2005). Factor Analysis Methods. In R. A. Swanson \& E. F. I. Holton (Eds.), Research in Organizations: Foundations and Methods of Inquiry (pp. 181-199). Berrett-Koehler Publishers, Inc.

Yeung, R. R. (1996). The acute effects of exercise on mood state. Journal of Psychosomatic Research, 40(2), 123-141. https://doi.org/https://doi-org.libproxy.wustl.edu/10.1016/00223999(95)00554-4

Zimmerman, J., Woods, W. C., Ritter, S., Happel, M., Masuhr, O., Jaeger, U., \& Spitzer, C. (2019). Integrating structure and dynamics in personality assessment: First steps toward the development and validation of a Personality Dynamics Diary. Psychological Assessment, 31(4), 516-531. https://doi.org/10.31234/osf.io/5zcth 


\section{Table 1.}

Correlations between ESM items and self-report measures at baseline

\begin{tabular}{llc}
\hline ESM item & Corresponding trait level measure & Correlation \\
\hline How down do you feel right now? & BDI - II: Sadness & .13 \\
How happy do you feel right now? & PANAS: Positive affect mean composite & .25 \\
How calm do you feel right now? & BDI-II: Agitation & -.12 \\
How irritated do you feel right now? & BDI-II: Irritation & .08 \\
How anxious do you feel right now? & BDI-II: Agitation; LSAS: Social anxiety & $-.10,-.06$ \\
& mean composite & -.13 \\
How lonely do you feel right now? & UCLA-R: Loneliness mean composite & -.09 \\
How accomplished do you feel right now? & BDI-II: Past failure & .12 \\
Are you currently avoiding a social & LSAS: Social avoidance mean composite & -.09 \\
situation or interaction? & & $-.40 *$ \\
How drowsy are you right now? & BDI-II: Tiredness/fatigue & .10 \\
How pleased are you with your experience & BDI-II: Anhedonia & -.32 \\
right now? & & -.16 \\
How restless do you feel right now? & BDI-II: Agitation & .10 \\
How focused are you right now? & BDI-II: Concentration difficulties \\
How hungry do you feel right now? & BDI-II: Loss of appetite & \\
How physically active (e.g., walking, & BDI-II: Loss of energy & \\
exercising, stretching) have you been & & \\
since the last beep? & & \\
\hline Note: Corresponding trait level measures were administered as part of a separate study. ESM= Ecological sampling \\
*
\end{tabular}


Table 2.

Bivariate correlations between ESM items from multilevel model

\begin{tabular}{|c|c|c|c|c|c|c|c|c|c|c|c|c|c|c|}
\hline & 1. & 2. & 3. & 4. & 5. & 6. & 7. & 8. & 9. & 10 . & 11. & 12. & 13. & 14. \\
\hline \multicolumn{15}{|l|}{ 1. Down } \\
\hline 2. Нарpy & $-.53 *$ & & & & & & & & & & & & & \\
\hline 3. Calm & $-.56 * *$ & $.75 * *$ & & & & & & & & & & & & \\
\hline 4. Irritated & $.69 * *$ & $-.42 *$ & $-.42 *$ & & & & & & & & & & & \\
\hline 5. Anxious & $.66 * *$ & $-.40^{*}$ & $-.47^{*}$ & $.57 * *$ & & & & & & & & & & \\
\hline 6. Lonely & $.76^{* *}$ & $-.66 * *$ & $-.53 *$ & $.55 * *$ & $.53 *$ & & & & & & & & & \\
\hline 7. Pleased & $-.51 *$ & $.92 * *$ & $.65 * *$ & $-.36^{*}$ & $-.43 *$ & $-.66 * *$ & & & & & & & & \\
\hline 8. Drowsy & $.78 * *$ & $-34 *$ & $-.38^{*}$ & $.62 * *$ & $.56 * *$ & $.57 * *$ & -.30 & & & & & & & \\
\hline 9. Accomplished & $-.42 *$ & $.76^{* *}$ & $.69 * *$ & $-.40 *$ & $-.39 *$ & $-.43 *$ & $.83 * *$ & -.19 & & & & & & \\
\hline $\begin{array}{l}\text { 10. Social } \\
\text { Avoidance }\end{array}$ & $.46^{*}$ & -.15 & -.33 & $.49 *$ & .28 & $.35^{*}$ & -.20 & .33 & -.16 & & & & & \\
\hline 11. Restless & $.41^{*}$ & -.04 & $-.34^{*}$ & $.50^{*}$ & $.44^{*}$ & .22 & .01 & $.34 *$ & -.09 & $.42 *$ & & & & \\
\hline 12. Focused & $-.51 *$ & $.72 * *$ & $.65^{* *}$ & $-.47 *$ & $-.36^{*}$ & $-.47^{*}$ & $.71 * *$ & $-.35 *$ & $.83 * *$ & -.16 & -.16 & & & \\
\hline 13. Hungry & .26 & .06 & -.29 & .20 & .21 & .13 & .13 & .27 & .10 & .24 & .28 & .11 & & \\
\hline $\begin{array}{l}\text { 14. Physical } \\
\text { Activity }\end{array}$ & .20 & .16 & .04 & .15 & .05 & .19 & .23 & .27 & $.38^{*}$ & $.34 *$ & .29 & .28 & $.59 * *$ & \\
\hline
\end{tabular}


PERSONALIZED MODELS OF INTERNALIZING SYMPTOMS

Table 3.

Estimates from the multilevel model

\begin{tabular}{|c|c|c|c|c|}
\hline Path & $\begin{array}{c}\text { Std. } \\
\text { estimate }\end{array}$ & $\begin{array}{l}\text { Posterior } \\
\text { S.D. }\end{array}$ & $\begin{array}{l}\text { 95\% Credible interval, } \\
\text { Lower bound }\end{array}$ & $\begin{array}{r}95 \% \text { Credib } \\
\text { Upper }\end{array}$ \\
\hline Day & $0.04 *$ & 0.02 & 0.01 & $0 . \mathrm{C}$ \\
\hline Survey & 0.02 & 0.03 & -0.04 & $0 . \mathrm{C}$ \\
\hline Down & $0.18 *$ & 0.02 & 0.13 & 0.2 \\
\hline Happy & -0.04 & 0.07 & -0.09 & $0 . \mathrm{C}$ \\
\hline Calm & $-0.04 *$ & 0.02 & -0.08 & $0 . \mathrm{C}$ \\
\hline Irritable & 0.02 & 0.02 & -0.02 & $0 . \mathrm{C}$ \\
\hline Anxious & 0.04 & 0.02 & -0.01 & $0 . \mathrm{C}$ \\
\hline Lonely & $0.06^{*}$ & 0.02 & 0.02 & 0.1 \\
\hline Accomplished & -0.02 & 0.02 & -0.07 & $0 . \mathrm{C}$ \\
\hline Hungry & -0.02 & 0.02 & -0.05 & $0 . \mathrm{C}$ \\
\hline Physical Activity & $0.04 *$ & 0.02 & 0.002 & $0 . \mathrm{C}$ \\
\hline Social Avoidance & $0.04 *$ & 0.02 & 0.01 & $0 . \mathrm{C}$ \\
\hline Drowsy & 0.01 & 0.02 & -0.03 & $0 . \mathrm{C}$ \\
\hline Pleased & -0.002 & 0.03 & -0.05 & $0 . \mathrm{C}$ \\
\hline Restless & 0.01 & 0.02 & -0.03 & $0 . \mathrm{C}$ \\
\hline Focused & -0.003 & 0.02 & -0.04 & $0 . \mathrm{C}$ \\
\hline
\end{tabular}

Note. $*$ denotes a $95 \%$ credible interval that does not include 0 , suggesting statistical significance. 


\section{Figure 1.}

Statistically significant pathways from mutlilevel and idiographic models

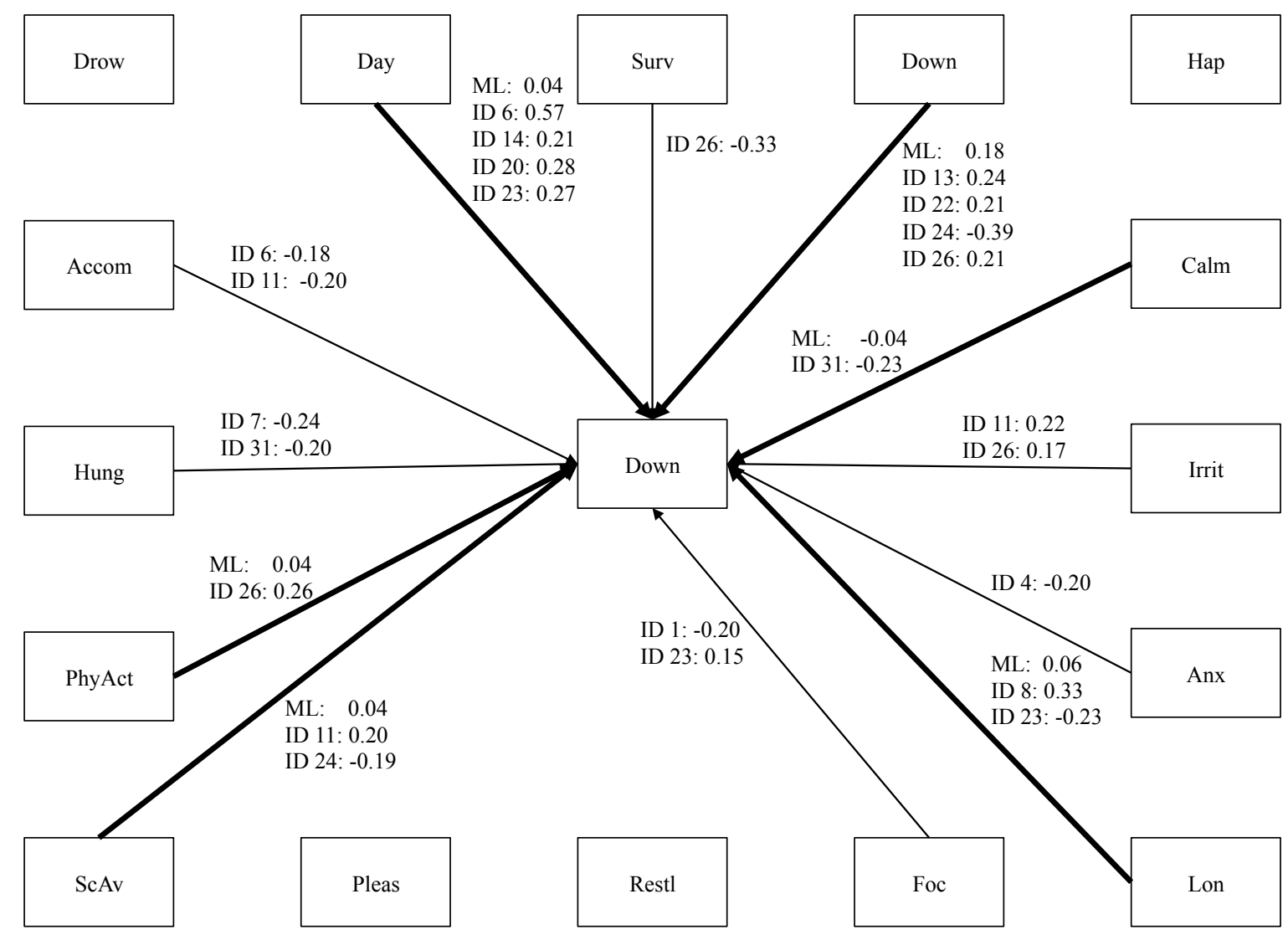

Note. Estimates from the multilevel model and individual models are listed by ID. Only standardized pathways with credible intervals that do not include 0 (indicating statistical significance) are shown here. Thicker lines indicate that the pathway was significant in the multilevel model. Surv = Survey; Hap = Happy; Irrit = Irritable; Anx = Anxious; Lon $=$ Lonely $;$ Foc $=$ Focused $;$ Restl $=$ Restless $;$ Pleas $=$ Pleased $;$ ScAv $=$ Social avoidance; PhAct $=$ Physical Activity; Hung = Hungry; Accom = Accomplishment; Drow = Drowsy 
Figure 2.

Overlap between multilevel and idiographic estimates
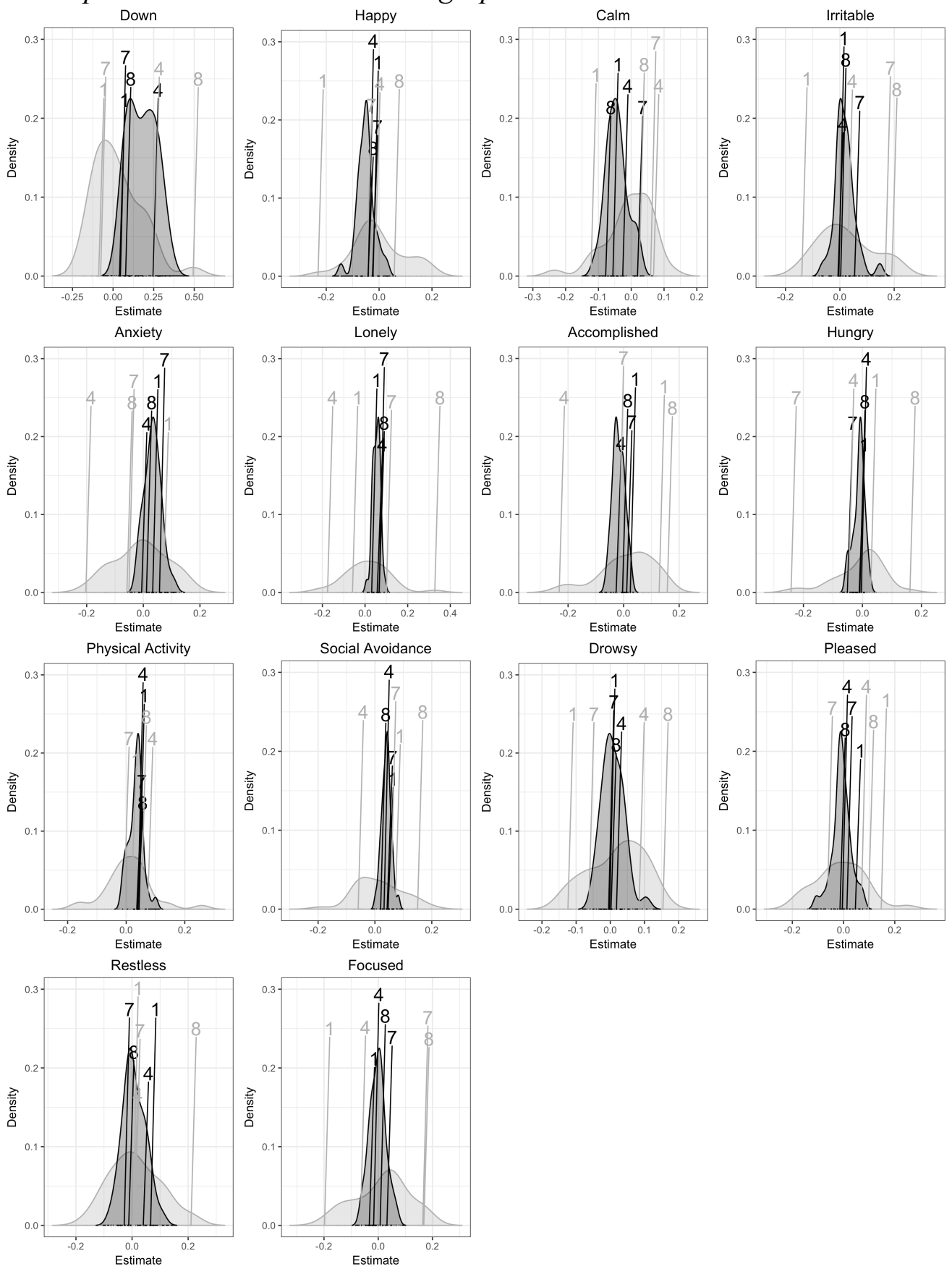

Note. The light grey density plot represents the individual-level estimates for each ESM pathway predicting down from the individual-level models. The dark grey density plot represents the individual-level estimates for each ESM pathway predicting down from the multilevel model. Participants with fewer than 100 time points (IDs 1, 4, 7, and 8) are noted. 